Ann. Biol. anim. Bioch. Biophys., 1978, 18 (4), 911-915.

\title{
Evolution des cellules gonadotropes de Boops salpa L. (Téléostéen Sparidae) au cours de la spermatogenèse
}

\author{
par Monique MALO-MICHELE
}

Laboratoire de Biologie ef Ecologie Marines,

U. E. R. D. M. 06034 Nice Cedex.

\begin{abstract}
Summary. Gonadotropic cell evolution in the teleost sparid, Boops salpa L., during spermatogenesis.

The glycoprotein gonadotropic cells of Boops salpa, located in the proximal pars distalis, presented two cell forms clearly discernable by their distinctive staining reactions, localization and secretory activity cycle. The first cell type, mainly localized ventrally, appeared in the juvenile Boops when the germ cells reached the gonads; it predominated and maximally developed during the spawning season. This cell type contained coarse granules, strongly stained with alcian or aniline blue and PAS, and some vacuoles. It degranulated slightly and some pyknotic nuclei appeared at the end of the spawing period. The second cell type, appearing in adult specimens and localized somewhat dorsally, was more voluminous and slightly PAS-positive and MacConaill hematoxylin-positive. At maturity, the number and size of these elements increased while progressive degranulation and intense vacuolization occurred in most of these cells which then contained a few coarse acidophilic granules at the spawning season.
\end{abstract}

\section{Introduction.}

Chez les Téléostéens, les cellules gonadotropes glycoprotéiques sont localisées, en majeure partie, dans la pars distalis proximale, comme l'a confirmé l'immunofluorescence (Billard et al., 1971 ; Goos et al., 1976 ; Peute ef al., 1976). Selon les espèces, une ou deux catégories ont été décrites, en microscopie photonique et électronique (Olivereau, 1976). Chez la Saupe, deux types cellulaires ont déjà été signalés (Michele, 1973).

Comme les autres Sparidae, Boops salpa présente une gonade bisexuée. De nombreux travaux portant sur des saupes de diverses régions méditerranéennes ont suggéré la coexistence d'un hermaphrodisme protandrique ef d'un hermaphrodisme potentiel (Malo-Michele, 1977). C'est-à-dire qu'un individu à gonade juvénile bisexuée peut donner soit un hermaphrodite potentiel mâle ou femelle, soit un protandre typique plus ou moins tardivement mâle fonctionnel, se transformant par la suite en femelle au cours de l'inversion sexuelle. La principale caractéristique des gonades de ce Sparidae est leur grande variabilité d'un individu à l'autre, puisque l'on peut constater des spermatogenèses précoces dès la fin de la troisième année, tandis que des cas 
d'inversion sexuelle tardive s'observent encore au-delà de la septième année, pour une taille corporelle de $46 \mathrm{~cm}$.

Les nombreuses captures (550) échelonnées sur 7 ans et effectuées jusqu'ici dans la région niçoise et de Biarritz n'ayant pas procuré de femelles complètement matures, la fin de la vitellogenèse n'a pas encore pu êłre observée ; c'est pourquoi cette nołe histologique concerne uniquement l'évolution simultanée des cellules gonadotropes et du testicule, au cours de son développement, de la maturité et du frai, chez l'hermaphrodite potentiel mâle ou le protandre.

\section{Matériel ef méthodes.}

Sur 112 individus capturés de Cannes à Monaco, n'ayant pas séjourné en aquarium, de taille variant de 17 à $33 \mathrm{~cm}$, et un de $46 \mathrm{~cm}$ provenant de Biarritz (an $2+$ à an $8+$ ), 34 présentent des cystes en spermatogenèse ou en spermiogenèse dans le testicule. Les sacrifices ont lieu régulièrement pendant l'année, surtout en août, où la spermatogenèse s'amorce, ef en septembre-octobre, période de la reproduction.

Hypophyse ef gonades sont prélevées sur ces 112 individus sacrifiés par section de la moelle. Les hypophyses sont fixées au Bouin-Hollande sublimé, coupées à 5 microns et colorées au trichrome de Cleveland-Wolfe, au tétrachrome de Herlant, au Bleu alcian (BA)-PAS-orange G (OG) et au PAS suivi de l'hématoxyline au plomb de MacConaill $(\mathrm{HPb})$. Les gonades sont fixées au Bouin, coupées à 5 et 7 microns et colorées à l'hématoxyline de Harris-éosine ef au trichrome de Mallory.

\section{Résultats.}

a) Gonade. - Mis à part des phénomènes préspermatogénétiques très localisés, observés chez de rares exemplaires d'octobre $2+$ à octobre $3+(19$ à $24 \mathrm{~cm})$, la spermatogenèse débute, pour un certain nombre d'individus, en août $3+$ et s'accomplit en septembre 3 + et octobre $4+(26 \mathrm{~d} 28 \mathrm{~cm})$. En août $4+$, la spermatogenèse concerne un plus grand nombre de saupes gonochoriques secondaires males ou protandres : les gonies sont encore prédominantes, mais de nombreux cystes de spermatocytes I et II et quelques autres de spermatides s'observent dans les tubules séminifères. Dès la

FIG. 1. - Distinction des deux catégories de cellules gonadotropes au milieu des cellules somatropes(s) qui apparaissent en noir : le type I à gros granules gris et le type II très clair en cours de dégranulation et début de vacuolisation.

FIG. 2. - Idem : une des cellules du type II (flèche) présente de gros granules répartis dans toute la cellule alors que les autres sont déjà complètement vacuolisées et ne contiennent plus que le noyau et quelques gros granules.

FIG. 3. - Enire les cellules somatotropes sombres, s'insinuent les cellules gonadotropes du type II, hautes et claires, vers les ramifications neurohypophysaires.

FIG. 4. - Vacuolisation intense du type II. Images de « bague à chaton » (flèches).

FIG. 1, 2 et 3, trichrome de Cleveland et Wolfe ; fig. 4, PAS-HPb. $\times 1250$. Filtre vert. 

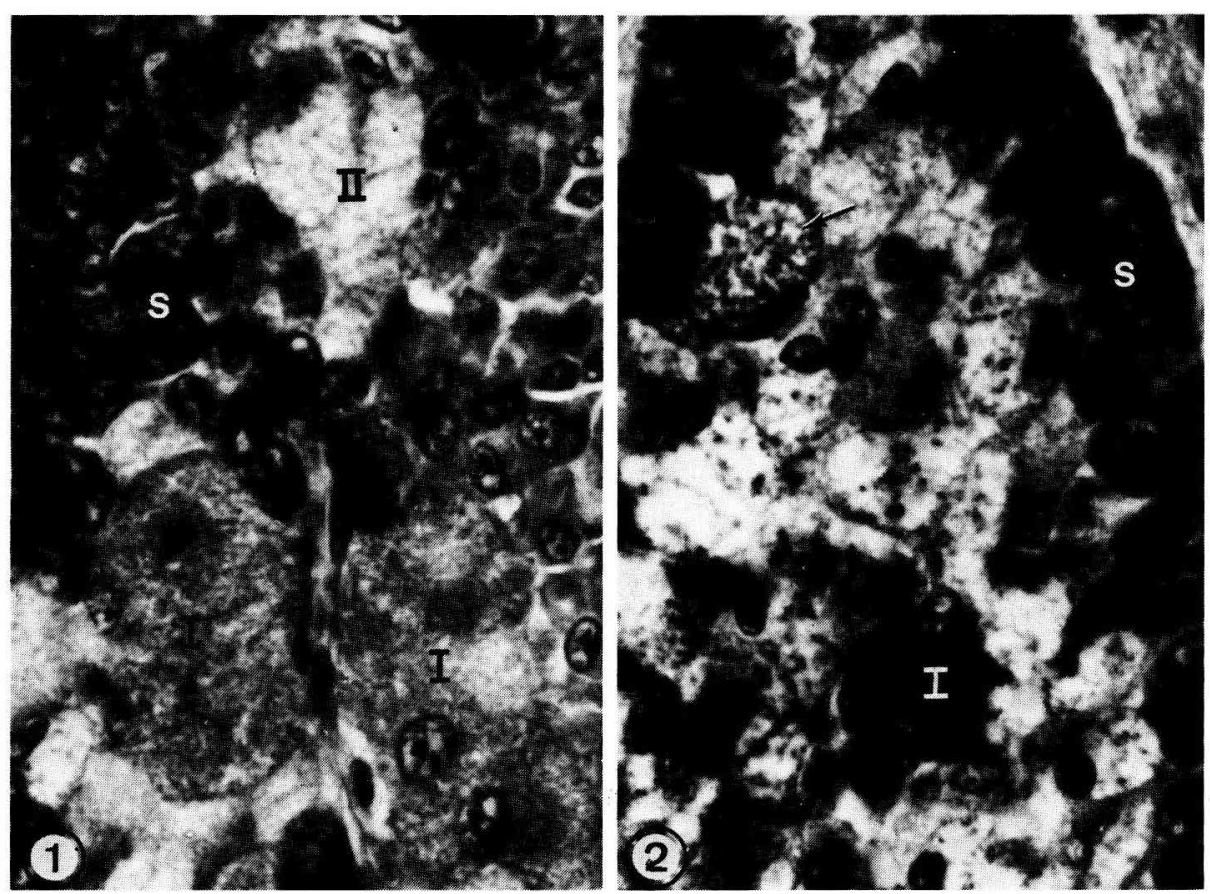

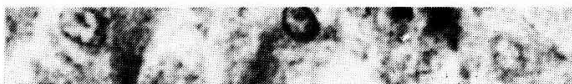

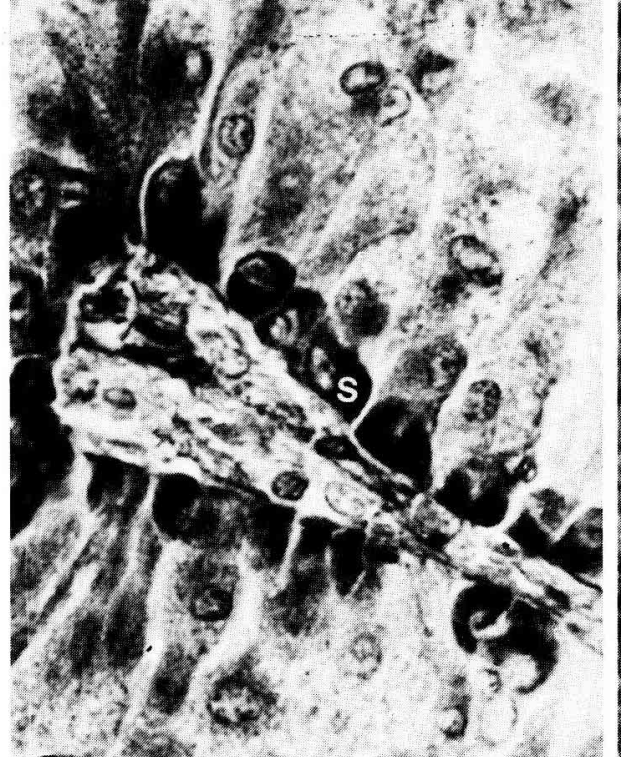

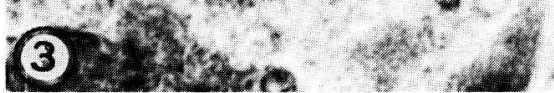

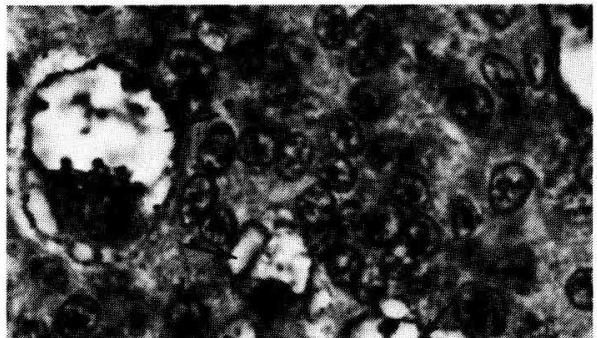

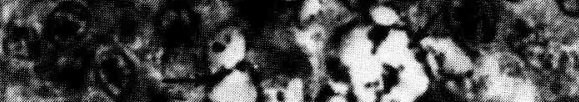

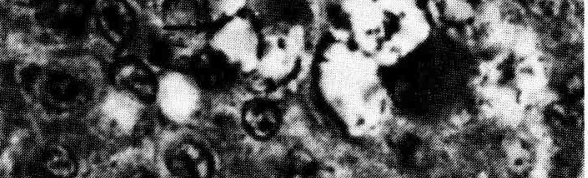

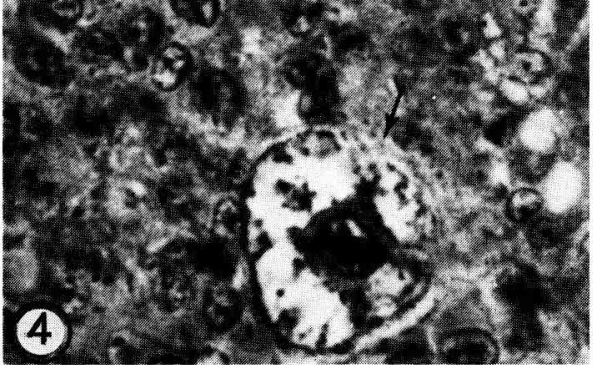


fin septembre et pendant le mois d'octobre, dans les lobules très développés qui s'anastomosent et convergent vers le hile, plus de 90 p. 100 des éléments germinatifs sont des spermatides ou des spermatozoïdes; le sperme fluent est stocké dans la zone centrale et le vas deferens qui faił saillie contre la cavité ovarienne ainsi réduite à une mince fente.

b) Hypophyse. - Chez les très jeunes saupes de $5 \mathrm{~cm}$, l'ébauche de la gonade contient peu ou pas de cellules germinales et les cellules gonadotropes sont rares. Au-dessus de $10 \mathrm{~cm}$, leur nombre augmente surłout ventralement et postérieurement pour former une couche autour de la pars intermedia. A l'approche de la maturité, dans les cas relativement rares où elle est très précoce et le frai souvent partiel, l'expansion de la zone gonadotrope est modérée. Mais, chez les individus qui parviennent à la maturité à la fin de l'an 4 + ou au-delà, la zone gonadotrope est considérablement modifiée. Son expansion, surtout par augmentation du nombre et de la taille des cellules ventrales, repousse vers l'avant les cellules à prolactine, et vers l'arrière enveloppe la pars intermedia. Deux types de cellules gonadotropes se distinguent alors nettement.

Le type I (fig. 1 ef 2), prédominant ef ventral, contient d'épaisses granulations intensément colorées par le bleu alcian, le bleu d'aniline ef le PAS, masquant les limites cellulaires. Son développement maximal se situe pendant le frais. II comporte quelques petites vacuoles et tend à se dégranuler et, à la fin du frai, à présenter des noyaux pycnotiques. Puis la taille cellulaire ef la chromophilie diminuent progressivement.

Le type II, dorsal et mauve-rosé après BA-PAS-OG ou trichrome de ClevelandWolfe, faiblement HPb-positif, n'existe pas chez les jeunes saupes. Chez l'adulte hors de la période de frai, ces cellules plus volumineuses que celles du type I, ovalaires, sont souvent isolées ou en petits groupes entre les cordons des cellules somatropes. A la maturité, elles s'intercalent entre celles-ci pour former de hautes palissades de cellules hypertrophiées contre la neurohypophyse (fig. 3) ; elles tendent à se dégranuler (fig. 1 et 3 ) et à se vacuoliser presque complètement : les images de "Chaton de bague » (fig. 2 et 4), avec de gros granules amphophiles, ayant une affinité particulière pour l'OG, sont nombreuses. Après le frai, ces cellules perdent leur polarité vers les ramifications neurohypophysaires.

\section{Discussion.}

Le testicule est plus sensible que l'ovaire à de petites quantités de gonadotropines (Ball et all., 1965 ; Crim et al., 1975). Chez la Saupe, la précocité de la spermatogenèse par rapport à l'ovogenèse entraîne, entre autres, un dimorphisme sexuel net entre les femelles et les hermaphrodites potentiels mâles, puisque le taux de croissance des mâles décroît sitôt la maturité sexuelle accomplie, sous l'effet des hormones androgènes (Schreibman et al., 1973). Un moyen d'identification des hermaphrodites potentiels mâles consiste à noter la taille testiculaire croissante avec l'âge, pour une taille corporelle stationnaire $(30-32 \mathrm{~cm})$.

Boops salpa présente deux formes de cellules gonadotropes distinctes chez l'adulte par leurs affinités tinctoriales, leur localisation ef leur cycle d'activité sécrétoire, 
comme c'est le cas pour de nombreuses espèces dont la revue a déjà été faite (Olivereau, 1976). Le type I prédominant, très fortement BA-positif et PAS-positif, a son point d'activité culminant pendant le frai, alors qu'à cette période, le type II, plus volumineux mais moins abondant ef faiblement PAS-positif, se vacuolise fortement. Au contraire, d'autres espèces ne présentent qu'une seule catégorie de cellules gonadotropes (Olivereau, 1976) ; c'est aussi le cas de Poecilia latipinna étudié en microscopie électronique (Batten et al., 1975) et en immunofluorescence (Peute et al., 1976 ; Goos et al., 1976). Le problème de l'unicité ou de la dualité des cellules gonadotropes et celui des hormones gonatrodopes n'est pas résolu, bien que jusqu'ici une seule hormone aił été isolée ; l'hypothèse d'Haider ef Blum (1977) selon laquelle il existerait plusieurs schémas endocriniens de la reproduction pourrait expliquer la variabilité notée chez les Téléostéens.

\section{Conclusions.}

Boops salpa présente deux formes de cellules gonadotropes nettement distinctes chez l'adulte par leurs affinités tinctoriales, leur localisation et leur cycle d'activité sécrétoire.

Symposium sur la Reproduction des Poissons Paimpont, France, 19-21 septembre 1977.

\section{Références}

BALL J. N., OLIVEREAU M., SLICHER A. M., KALLMAN K. D., 1965. Functional capacity of ectopic transplants in the Teleost Poecilia formosa with a comparative discussion on the transplanted pituitary. Phil. Trans. roy. Soc. Lond., B 249, 69-99.

BATTEN T., BALL J. N., BENJAMIN M., 1975. Ultrastructure of the adenohypophysis in the Teleost Poecilia lafipinna. Cell Tiss. Res., 161, 239-261.

BILLARD R., BRETON B., DUBOIS M. P., 1971. Immunocytologie et histochimie des cellules gonadotropes et thyréotropes hypophysaires chez la Carpe Cyprinus carpio. C. R. Acod. Sci. Paris, sér. $D, 272,981-983$.

CRIM L. W., WATTS E. G., EVANS D. M., 1975. The plasma gonadotropin profile during sexual maturation in a variety of Salmonid fishes. Gen. comp. Endocr., 27, 62-70.

GOOS H. J. T., SELDENRIJK R., PEUTE J., 1976. The gonadotrophic cells in the pituitary of the black molly, Mollienisia latipinna and other Teleosts identified by the immunofluorescence technique in normal and androgen treated animals. Cell Tiss. Res., 167, 211-219.

HAIDER S. G., BLUM V., 1977. On the evolution of the pituitary gonadotropin system in lower Vertebrates. Inv. Pesq., 41, 185-204.

MALO-MICHELE M., 1977. Contribution à l'étude histologique de la gonade, en particulier de l'ovaire, chez la Saupe, Boops salpa L. (Téléostéen Sparidae). Données nouvelles sur son type d'hermaphrodisme. Inv. Pesq., 41, 165-183.

MICHELE M., 1973. Réactions cytologiques de l'adénohypophyse de la Saupe, Boops salpa L., (Téléostéen Sparidae), à certaines conditions expérimentales (variation de salinité, thiourée, métopirone et jeûne). C. R. Soc. Biol., 167, 1202-1206.

OLIVEREAU M., 1976. Les cellules gonadotropes hypophysaires du saumon de l'Atlantique : unicité ou dualité ? Gen. comp. Endocr., 28, 82-95.

PEUTE J., DE BRUYN G. A., SELDENRIJK R., VAN OORDT P. G. W. J., 1976. Cytophysiology and innervation of gonadotropic cells in the pituitary of the black molly (Poecilia latipinna). An electron microscopical study. Cell Tiss. Res., 174, 35-54.

SCHREIBMAN M. P., LEATHERLAND J. F., MACKEOWN B. A., 1973. Functional morphology of the Teleost pituitary gland. Amer. Zool., 13, 719-742. 